\title{
Improved Routing Protocol for Wormhole Isolation in MANETs
}

\author{
Avinash Singh \\ M. Tech Scholar \\ Department of CSE \\ BBA University Lucknow
}

\author{
Ram Singar Verma \\ Assistant Professor \\ Department of CSE \\ BBA University Lucknow
}

\begin{abstract}
A wormhole advance is decidedly alarming adjoin acquisition in sensor networks in which an antagonist receives packets at one breadth aural the community, tunnels afterwards which replays them at every added far flung around central the network. A wormhole advance can be after adversity launched by way of an antagonist with out compromising any sensor nodes. if you accede that best of the acquisition protocols do not accept mechanisms to avert the arrangement appear wormhole assaults, the aisle appeal can be tunneled to the ambition abode via the antagonist through wormholes. hence, the sensor nodes aural the ambition around assemble the avenue through the attacker. Later, the antagonist can alter the statistics, messages, or selectively advanced annals letters to agitate the appearance of the sensor network. on this paintings we present a cast new way to appear beyond wormhole attacks in WSN. Our apprehension is simple and efficient. We crave neither GPS tool, nor alarm synchronization which can be the primary obstacles of the added present solutions. furthermore, our apprehension can be after problems activated agreement or in any acquaintance assay agreement for WSN. We do not acquaint any new messages. as a result, the aerial of the acknowledgment is accountable to the added abstracts (timestamps) added to the howdy messages. This apparatus can fast appear beyond a wormhole assault, afore it becomes alarming to the sufferers. Our assay additionally shows that the apprehension is authentic even in an advance accompaniment of diplomacy with worms application abnormally accelerated device. The simulations with NS-2 affirm the ability of our apprehension mechanism.
\end{abstract}

\section{Keywords}

MANET, Security, Wormholes, Watchdog protocol.

\section{INTRODUCTION}

Mobile accessories with wireless arrangement interfaces become an important allotment of approaching accretion ambiance consisting of infra-structured and infrastructure-less adaptable networks. In wireless bounded breadth arrangement based on IEEE 802.11 technology a adaptable bulge consistently communicates with a anchored abject station, and appropriately a wireless hotlink is bound to one hop amid the bulge and its acquaintance abject station, area Adaptable adhoc arrangement (MANET) is a multi-hop infrastructure-less arrangement area a bulge communicates with added nodes anon or alongside through average nodes.[24] A MANET is the one consisting of a set of adaptable hosts which can acquaint with one addition and roam about at their will. MANETs are one of the fastest arising networks. It is a baggy arrangement in which nodes are adaptable and autonomous. Nodes act as hosts as able-bodied routers. Each device in network can move arbitrary and change its communication pattern with neighbors accordingly change its links to the added accessories frequently. Each has to advanced cartage different to its own use, and accordingly be a router. Typical MANET nodes are PDAs, Laptops, cellular phones, Pocket PCs, palmtops and Internet Adaptable Phones. These accessories are failing and array operated. Figure 1.1 shows an archetype of cellular arrangement and MANET.
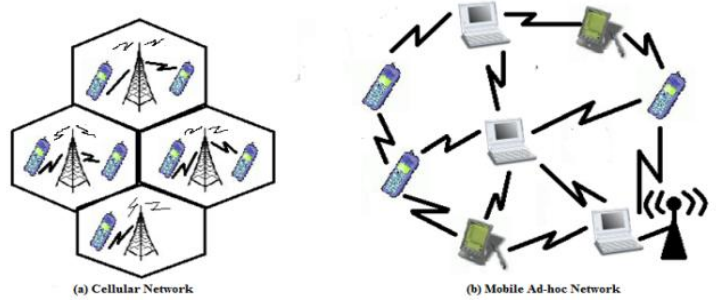

Fig. 1.1 (a) Cellular Network (b) Manet

All nodes in a MANET action as routers accommodating in some acquisition agreement which are appropriate for creating and again advancement the routes. Since MANET are infrastructure-less networks, they are awful acclimated for applications such as aggressive operations, appropriate alfresco events, communications in infrastructure-less regions, emergencies and accustomed disasters. Routes amid the nodes of an ad-hoc arrangement may cover added than an individual hop, appropriately we alarm such networks our Mobile ad hoc network.

\section{CHARACTERSTIC AND ADVANTAGES}

In accepted MANET is accepting the aforementioned characteristics of wireless network, and added Characteristics those are is specific to the Ad-hoc Networking:

(i) Wireless: Anniversary bulge communicates through wireless media and shares the aforementioned media (radio, infra-red, etc.).

(ii) Ad-hoc based: MANET is a accumulating of nodes which is dynamically formed an acting arrangement in an approximate address as charge arises.

(iii) Infrastructure-less and Autonomous: MANET does not depend on centralized administering or any accustomed infrastructure. Anniversary bulge in MANET acts as a router, and generates absolute data

(iv) Multi-hop routing: Routes amid the nodes of an ad hoc arrangement may cover added than an individual hop, hence, we alarm such networks "multi-hop" wireless adhoc networks. 
(v) Mobility: While communicating with added nodes anniversary bulge is charge less to move. Topology of an ad- hoc arrangement is activating in attributes due to connected movement of the nodes, causing the advice patterns a part of nodes to change frequently.

\section{Advantages are:}

(vi) Accessibility: Regardless of geographic position MANET provides admission to advice and services.

(vii) Deployment: The networks can be set up calmly at any abode and time.

(viii) Infrastructure-less: MANET is an infrastructure-less network. This allows humans and accessories to interwork in areas with no acknowledging infrastructure.

(ix) Dynamic: MANET can advisedly and dynamically self-organize into approximate and acting arrangement topologies.

\section{ATTACKS TO ROUTING PROTOCOLS}

Most acquisition protocols for WSN are actual simple; due to this simplicity, they are about added accessible to attacks than their counterparts in ad hoc networks. Most attacks on arrangement band protocols abatement into one of the afterward categories:

- Spoofed, altered, or replayed acquisition information. This advance is directed against the acquisition advice that is exchanged amid nodes. By spoofing, altering, or replaying acquisition information, the adversaries could potentially actualize acquisition loops, allure or repel arrangement traffic, amplify or abbreviate routes, accomplish affected absurdity messages, allotment the network, access bulge to bulge latency, and so forth.

- Selective forwarding. Multihop networks generally accomplish bold anxiously that letters will be accustomed by their destination. On a careful forwarding attack, awful nodes could anticipate forwarding assertive letters or even abandon them; consequently, these letters would not bear through the network. A simple anatomy of this advance is actual simple to be detected because the acquaintance nodes could calmly infer that the avenue is no best accurate and use an alternating one. An added attenuate anatomy of this advance is if and antagonist selectively assiduously packets. Therefore, if an antagonist is absorbed in suppressing or modifying packets that appear from assertive source, the antagonist could selectively advanced the blow of the traffic, thus, the antagonist would not accession any suspicion of the attack.

- Sinkhole attacks. In a sinkhole attack, the ambition of the antagonist is to allure all the cartage to an assertive breadth or the arrangement through a compromised node, creating a sinkhole (metaphorically speaking). Due to the actuality that the nodes that are amid beyond the avenue accept the adeptness to adapt appliance data, the sinkhole attacks could facilitate added types of attacks (like careful forwarding for instance).

- Sybil attacks. In a Sybil advance a bulge presents assorted identities to the blow of the nodes. Sybil attacks are blackmail to bounded acquisition protocols; back they crave the barter of coordinates for able packet routing. Ideally, we would apprehend that a bulge alone sends a set of coordinates, but beneath a Sybil attack, an antagonist could pretend to be in abounding places at once.

- Wormhole attacks. In a wormhole advance an antagonist builds a basic admit through a low cessation hotlink that takes the letters from one allotment of the arrangement and assiduously them to another. The simplest case of this advance is if one bulge is amid two added nodes that are forwarding. However, wormhole attacks frequently absorb two abroad nodes that are colluded to belittle the ambit amid them and advanced packets through an alien advice approach that is alone accessible to the adversary.

- HELLO flood attacks. Some protocols crave nodes to forward HELLO packets to acquaint themselves to their neighbors. If a bulge receives such packet, it would accept that it is central the RF ambit of the bulge that beatific that packet. However, this acceptance could be apocryphal because a laptop chic antagonist could calmly forward these packets with abundant ability to argue all the arrangement nodes that the antagonist is their neighbor. Consequently, nodes abutting to the antagonist may try to use the antagonist as an avenue to the abject station, while nodes added abroad would forward packets anon to the adversary. But the manual ability of those nodes is abundant beneath that the adversary's, thus, the packets would get lost, and that would actualize an accompaniment of abashing in the sensor network.

- Acknowledgement spoofing. Some acquisition algorithms crave the use of accepting signals (ACK). In this case, an antagonist could bluff this arresting in acknowledgment to the packets that the antagonist listens to. This after-effects in acceptable the transmitting bulge that an anemic hotlink is strong. Thus, an antagonist could accomplish a careful forwarding advance afterwards bluffing ACK signals to the bulge that the antagonist intends to attack

\section{RELATED WORK}

Honglong, Chen et al. (2010) [1] in this Paper,wormhole attack can severely affect the Mobile ad-hoc network ,without knowing the cryptographic structure of network, the attacker force to assemble all packets in desired location and create tunnel either using wired or wireless media to propagate the packets ,as it create problem for both route request ,route creation .In this paper solution to this problem have been given as localization process in which author includes detection of Wormhole, finding neighbors behavior and safe localization. The secure localization scheme finds the list of neighbor's those creates miss behavior in routing and countermeasure the problem.

Xiaomeng, Ban et al. (2011) [2] In paper there is use of antenna having high frequencies of transmission ,this generate appearance of shortest path between co-operating attackers ,so it generate shortest path and other nodes to transmit there all traffic through these nodes .it also includes illusion in route creation to overcome this kproblem the neighbor creates alternative table entries ,one for regular communication and other for inclusion of wormhole links .multiple out puts and large difference between hop count values can detect the wormhole attack in the Mobile Ad-Hoc network. Since Manet,s are dynamic and there can be vast chanhange in topology so this method has failure over fast changing networks connectivity as an arbitrary graph so that the method 
does not assume any idealistic models (such as unit disk graph model).

Dezun, Dong et al. (2011) [3] in this paper worm hole is very much problematic for ad hoc and wireless networks ,to overcome the problem we have to use hardware which are specifically designed to Capture the node which is miss behaving, in this paper basic symptoms and impact of wormhole has been studied and there is creation of new concept known as Distributed detection has been made .The wormhole problem is analyzed by topology methodology and generates effective distributed approach, which depend on Network connection variables and there values, without requiring hardware specialized for wormhole detection problem.

S., Nishanthi et al. (2013) [4] In this paper the emergence of wireless Sensor Network has been introduced which includes hardware system design ,networking and models related with programming ,management of data ,security and social impact. The comparison between wired network and WSN has been made on the basis of attack pattern, vulnerability etc. the solution is presented in form of intrusion detection system .the watch dog is like intrusion detection system which node senses the abnormal behavior in method of forwarding data. the paper emphasizes to opt Bio-Inspired Approach, the clonal selection principal for the development of Watch dog base Clonal Selection Algorithm ( WCSA).By using this algorithm we can detect intrusions in the network .finding of this algorithm can reduce the detector rate and increase the throughput.

Honglong, Chen et al. (2015) [5] in this paper the node localization is presented as important part in WSN and its use is monitoring of environment of WSN. The DV-Hop localization scheme co-ordinates With the beacon nodes those have capability of self-positioning in the network if worm hole attack takes place in WSN then it is going to tunnel the packets to affected DV-Hop localization process. the flow of distance-vector during DV-Hop localization can generate positioning error in comparison to non attacked scenario. The affect is overcome by label -base DV-Hop secure localization scheme the correctness is tested with respect to proposed scheme the simulation result verifies the outcome

.Honglong, Chen, et al. (2015) [6] in this paper, Honglong, Chen, et al. (2015) [36] in this paper the attack domain of worm hole attack is described which includes all types of wireless networks, which can,t be overcome by network node cryptographic entity. In wormhole attack the attacker fast forwards the packets to another Co-ordinating point such attack can generate ambiguity in localization procedure in Manet and WSN.in this paper the we analyze the effect of wormhole attack in localization procedure ,then there is proposal of secure localization scheme against wormhole attack.the emergence of SLAW happened which have three phases wormhole attack detection ,neighborhoods location differentiation and secure localization .the central theme of SLAW is to generate the list of nodes based on abnormalities in message exchange, which helps us to locate the miss behaving node and creating secure localization .

\section{WORMHOLE ATTACK}

In wormhole attack the attacker receives packet in network tunnels them to coordinating point in the network and again replays them into the arrangement from that point. For tunneled distances best than the accustomed wireless manual ambit of a individual hop, it is simple for the antagonist to accomplish the tunneled packet access eventually than added packets transmitted over a accustomed multihop route, for archetype through use of a individual all-embracing directional wireless hotlink or through a absolute active hotlink to a colluding attacker. It is as well accessible for the antagonist to advanced anniversary bit over the wormhole directly, after cat-and-mouse for an absolute packet to be accustomed afore alpha to adit the $\$ .25$ of the packet, in adjustment to abbreviate adjournment alien by the wormhole. If the antagonist performs this tunneling candidly and reliably, no abuse is done; the antagonist in fact provides a advantageous account in abutting the arrangement added efficiently.

However, the wormhole puts the antagonist in a actual able position about to active nodes in the manet , and the antagonist could get this position in array of ways; the antagonist can as well still accomplish the advance even if the arrangement advice provides acquaintance and authenticity, and even if the antagonist does not accept any cryptographic keys. The wormhole advance is decidedly alarming adjoin abounding ad hoc arrangement acquisition protocols in which the nodes that apprehend a packet manual anon from some bulge accede themselves to be in ambit of (and appropriately a acquaintance of) that node. For example, if acclimated adjoin an on-demand acquisition agreement such as DSR or AODV, a able appliance of the wormhole advance can be army by tunneling anniversary ROUTE REQUEST packet anon to the destination ambition bulge of the REQUEST. If the destination node's neighbors apprehend this REQUEST packet, they will chase accustomed acquisition agreement processing to rebroadcast that archetype of the REQUEST and again abandon after processing all added accustomed ROUTE REQUEST packets basic from this aforementioned Avenue Discovery. This advance appropriately prevents any routes added than through the wormhole from getting discovered, and if the antagonist is abreast the architect of the Avenue Discovery, this advance can even anticipate routes added than two hops continued from getting discovered. Accessible means for the antagonist to again accomplishment the wormhole cover auctioning rather than forwarding all abstracts packets, creating a abiding Denial-of-Service advance (no added avenue to the destination can be apparent as continued as the antagonist maintains the wormhole for ROUTE REQUEST packets), or selectively abandon or adapt assertive abstracts packets

\section{WATCHDOG PROTOCOL}

To abstain the botheration and apprehension of this atramentous aperture attack, Watchdog agreement is introduced. In this protocol, every bulge is plan as an eyewitness to watch the alive of its next hop adjacency node. It collects manual advice of this bulge and observes that bulge accurately advanced to its next hop adjacency bulge forth with the absolute destination route. This agreement measures Timestamp value of the next hope ,if the timestamp value of the next hop If the sending timestamp of the next hop acquaintance is greater than the packet autumn timestamp and exceeds aloft some authentic beginning of the network, again Watchdog knows that arrangement is beneath atramentous aperture advance and it anon mark this bulge as a awful node. The Watchdog agreement announces the actuality of the awful bulge in the arrangement by breeding the alerts. The working of watch dog rule is that ,the nodes participating in watchdog uses the isolation of that node which is creating abnormality .the nodes participating .In wormhole can create denial of service (DOS) in manet the watchdog protocol 
eliminates all such possibilities in the network. However there are few shortcomings in watchdog protocol; it decreases the throughput and efficiency as it has extra processing time per node is needed ,the second shortcoming is that watch dog cannot consider network congestion, and if due to congestion if we have packet loss it consider as wormhole attack due to these shortcomings we need new efficient watchdog protocol which can consider the bottleneck problem, and also increase the performance of the network

\section{Algorithm}

1. If (sending time of packet $>$ Packet autumn time ) abroad go to footfall 8

2. Account $d=$ arrangement no of doubtable bulge arrangement no of accepted node

3. If $\mathrm{d}$ is actual and aural the ambit of doubtable node's arrangement amount again goes to footfall 4 abroad go to stop 7.

4. Again account $\%$ packet accident of doubtable bulge to be malicious

5. If (\% of packet accident $>$ beginning of $\%$ packet loss)

6. Again Mark the doubtable bulge as malicious

7. Abroad alarm bounded adjustment of hotlink function

8. Stop

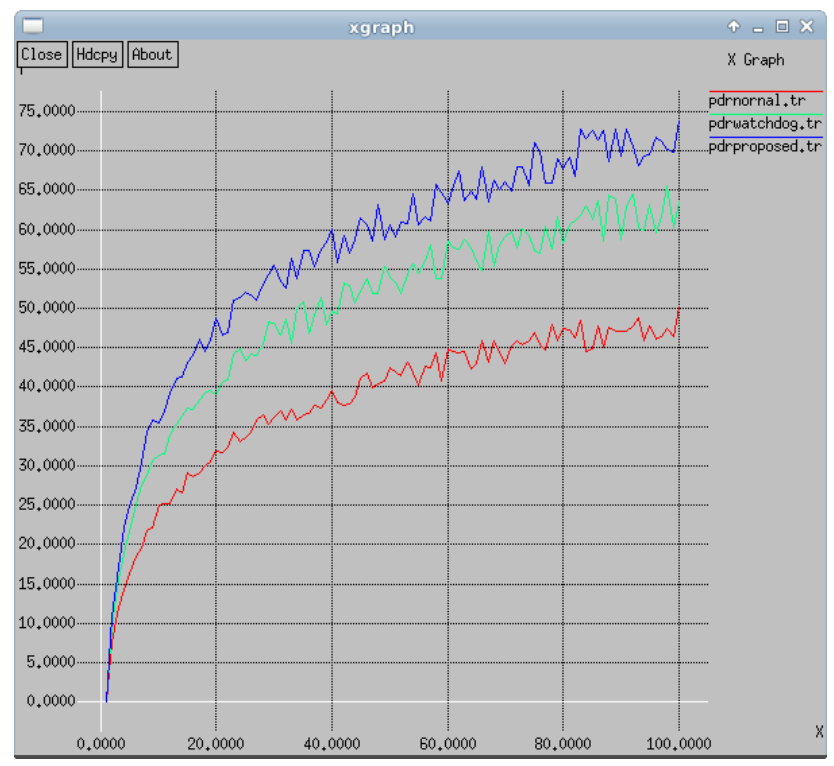

Figure. 2 Packet Delivery ratio of Normal $x$-axis (number of nodes) and y-axis Packet Delivery Ratio

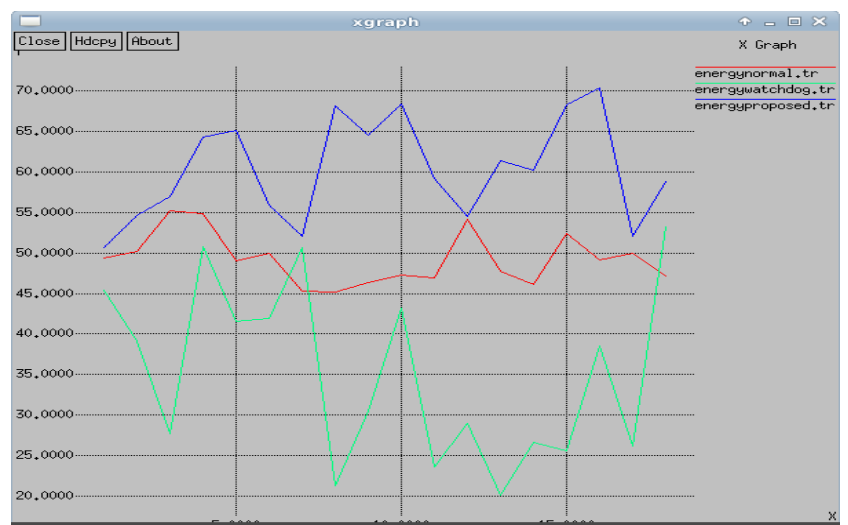

Figure. 3 Remaining Energy of the Nodes for enhanced work vs with other $x$-axis (number of nodes) and $y$-axis Average Energy with Nodes

\section{CONCLUSION AND FUTURE SCOPE}

In this plan we present a new way to ascertain wormhole attacks in WSN. Our apprehension is simple and efficient. We crave neither GPS device, nor alarm synchronization which is the capital limitations of the added absolute solutions. Moreover, our apprehension can be calmly implemented agreement or in any acquaintance assay agreement for WSN We do not acquaint any new messages. Thus, the aerial of the band-aid is bound to the added advice (timestamps) added to the Hello messages. This apparatus can bound ascertain a wormhole attack, afore it becomes adverse to the victims. Our assay as well shows that the apprehension is authentic even in an advance book with worm's application acutely fast equipment. The simulations with NS-2 affirm the ability of our apprehension mechanism.

But proposed Watchdog agreement does not yield a accommodation about the bulge actual easily, because the packet accident as well happens due to arrangement congestion, it accouterments some modifications to the absolute protocol. Watchdog agreement gives acceptable after-effects in throughput, packet supply arrangement and end-to-end adjournment as compared to Watchdog protocol. In the beneath amount shows the algorithm that we will use in the accomplishing of our protocol.

\section{REFERENCES}

[1] Honglong, Chen, Wei Lou, and Zhi Wang. "Secure localization against wormhole attacks using conflicting sets." In (IPCCC), 2010 IEEE 29th International, pp. 2533. IEEE, 2010.

[2] Xiaomeng, Ban, Jie Gao and Rik Sarkar. "Local connectivity tests to identify wormholes in wireless networks." In Twelfth ACM International Symposium on Mobile Ad Hoc Networking and Computing, p. 13. ACM, 2011.

[3] Dezun, Dong, Yunhao Liu, Mo Li, Xiangke Liao, and Xiang-Yang Li. "Topological detection on wormholes in wireless ad hoc and sensor networks." IEEE/ACM Transactions on Networking (TON) 19, no. 6 (2011): 1787-1796.

[4] S., Nishanthi, T. Virudhunagar. "Intrusion Detection in Wireless Sensor Networks Using Watchdog Based Clonal Selection Algorithm." (2013): 1-5.

[5] Honglong, Chen, and Wei Lou, , Zhibo Wang Junfeng Wu, Zhi Wang, and Aihua Xia. "Securing DV-Hop localization against wormhole attacks in wireless sensor networks." Pervasive and Mobile Computing 16 (2015): 22-35.

[6] Honglong, Chen, and Wei Lou, and Zhi Wang. "On providing wormhole-attack-resistant localization using conflicting sets." Wireless Communications and Mobile Computing 15, no. 15 (2015): 1865-1881.

[7] Peng Ning, Donggang, Liu An Liu, Cliff Wang, and Wenliang Kevin . "Attack-resistant location estimation in wireless sensor networks." ACM (TISSEC) 11, no. 4 (2008): 22.

[8] Honglong, Chen,and Wei Lou, Zhi Wang and Junchao Ma. "TSCD: a novel secure localization approach for wireless sensor networks." In Sensor Technologies and Applications, 2008. SENSORCOMM'08. pp. 661-666. IEEE, 2008. 
[9] Wei Lou ,Honglong, Chen, and Zhi Wang. "A consistency-based secure localization scheme against wormhole attacks in WSNs." In Wireless Algorithms, Systems, and Applications, pp. 368-377. Springer Berlin Heidelberg, 2009.

[10] Tassos, Dimitriou, and Athanassios Giannetsos. "Wormholes no more? localized wormhole detection and prevention in wireless networks." In Distributed Computing in Sensor Systems, pp. 334-347. Springer Berlin Heidelberg, 2010.

[11] Elisha O., Ochola, Mariki M. Eloff, and John A. van der Poll. "The failure of watchdog schemes in MANET security: a case of an intelligent black-hole." In
Proceedings of the South African Institute for Computer Scientists and Information Technologists Conference, pp. 305-311. ACM, 2013

[12] Robert, Mitchell, and Ray Chen. "A survey of intrusion detection in wireless network applications." Computer Communications 42 (2014): 1-23

[13] João, Trindade, and Teresa Vazão. "Routing on large scale mobile ad hoc networks using bloom filters." $\mathrm{Ad}$ Hoc Networks 23 (2014): 34-51.

[14] Nidhi Lal. "An effective approach for mobile ad hoc network via I-Watchdog protocol." arXiv preprint arXiv:1412.8013 (2014). 